\title{
Nine Limit Cycles in a 5-Degree Polynomials Liénard System
}

\author{
Junning Cai $\mathbb{D}$, Minzhi Wei $\mathbb{D}$, and Hongying Zhu \\ Department of Information and Statistics, Guangxi University of Finance and Economics, Nanning, Guangxi 530003, China
}

Correspondence should be addressed to Minzhi Wei; xiaoyanxiong123@163.com

Received 8 July 2020; Revised 31 August 2020; Accepted 10 October 2020; Published 2 November 2020

Academic Editor: Shouwei Li

Copyright (c) 2020 Junning Cai et al. This is an open access article distributed under the Creative Commons Attribution License, which permits unrestricted use, distribution, and reproduction in any medium, provided the original work is properly cited.

In this article, we study the limit cycles in a generalized 5-degree Liénard system. The undamped system has a polycycle composed of a homoclinic loop and a heteroclinic loop. It is proved that the system can have 9 limit cycles near the boundaries of the period annulus of the undamped system. The main methods are based on homoclinic bifurcation and heteroclinic bifurcation by asymptotic expansions of Melnikov function near the singular loops. The result gives a relative larger lower bound on the number of limit cycles by Poincaré bifurcation for the generalized Liénard systems of degree five.

\section{Introduction}

Consider the following perturbed Hamiltonian system:

$$
\begin{gathered}
\dot{x}=H_{y}(x, y)+\varepsilon p(x, y, \delta), \\
\dot{y}=-H_{x}(x, y)+\varepsilon q(x, y, \delta),
\end{gathered}
$$

where $H(x, y)$ is a polynomial of degree $n+1, p(x, y, \delta)$ and $q(x, y, \delta)$ are polynomials of degree $n, \varepsilon$ is the sufficiently small perturbation parameter, and $\delta \in D \subset \mathbb{R}^{N}$ with $D$ compact. Taking $\varepsilon=0$, system (1) becomes a Hamiltonian system:

$$
\begin{aligned}
& \dot{x}=H_{y}(x, y), \\
& \dot{y}=-H_{x}(x, y),
\end{aligned}
$$

and system (1) is usually called a near-Hamiltonian system. We assume that the unperturbed system (2) has a family of periodic orbits $\left\{\Gamma_{h}\right\}$ defined by $\{(x, y) \mid H(x, y)=h\}$, and the family forms a periodic annulus. The inner boundary of the periodic annulus is a center which may be an elementary one or a nilpotent one, and the outer boundary of the periodic annulus is usually a homoclinic loop or a heteroclinic loop or a polycycle. Most periodic orbits are broken when system (2) is perturbed and only a finite number of periodic orbits persist as limit cycles of system (1), which is the so-called Poincaré bifurcation. The most efficient tool to study
Poincaré bifurcation of system (1) is the following first-order approximation of the Poincaré map:

$$
M(h, \delta)=\oint_{\Gamma_{h}} q \mathrm{~d} x-p \mathrm{~d} y
$$

which is a continuous integral over the continuous ovals $\left\{\Gamma_{h}\right\}$ of $H(x, y)$ and is usually called the first-order Melnikov function or Abelian integral. The zeros of $M(h, \delta)$ correspond to the limit cycles by Poincaré bifurcation for system (1) (see $[1-3])$. It should be noted that studying the maximal number of zeros of $M(h, \delta)$ is the topic of weak Hilbert's 16 th problem. We note that the original version of Hilbert's 16th problem asks the maximal number of limit cycles of a general polynomial system:

$$
\begin{aligned}
& \dot{x}=P_{n}(x, y), \\
& \dot{y}=Q_{n}(x, y) .
\end{aligned}
$$

Let $H(n)$ denote the maximal number of limit cycles of system (4) and $H^{*}(n)$ denote the maximal number of limit cycles of system (1). Most results on the lower bounds of $H(n)$ are obtained in the literature as we know, by studying $H^{*}(n)$. However, it should be noted that it is also very difficult to determine $H^{*}(n)$ for a given degree $n$. We refer the works [4] for the relatively new results on the lower bounds of $H^{*}(n)$. 
In order to reduce the difficulty, researchers usually study some special forms of system (1), such as

$$
\begin{aligned}
& \dot{x}=y, \\
& \dot{y}=g(x)+\varepsilon f(x) y,
\end{aligned}
$$

which is called Liénard system of type $(m, n)$ having the degree $n^{*}=\max \{n+1, m\}$, where $g(x)$ and $f(x)$ are polynomials of degrees $m$ and $n$, respectively, and $\varepsilon$ is positive and very small. Let $H(n, m)$ denote the maximal number of limit cycles of system (5) and $I\left(n^{*}\right)$ denote the maximal number of limit cycles of system (5) of degree $n^{*}$. Dumortier and Li studied four Liénard systems with different portraits of type $(3,2)$ in a series of papers [5-8] and gave the corresponding sharp bound of number of limit cycles by Poincaré bifurcation. The exact bounds of $H(4,3)$ and $H(5,4)$ for some special Liénard systems were reported in [9-14] and references therein. For results on $H(7,6)$ associated with symmetric system (5), the relatively new works are referred [15-17]. It is usually very difficult to give the exact bound; however, lots of lower bounds of $H(m, n)$ have been obtained by studying the number of limit cycles near the center, homoclinic loops, and heteroclinic loops (see [18]). In particular, Xu and Li [19] investigated a Liénard system of type $(5, n)$ and proved $H(5,2) \geq 3, H(5,4) \geq 5$, $H(5,6) \geq 10, H(5,8) \geq 10$. In this paper, we study a type $(5,4)$ Liénard system which has a polycycle consisting of a homoclinic loop and a heteroclinic loop:

$$
\begin{aligned}
& \dot{x}=y, \\
& \dot{y}=-\frac{1}{7} x(7 x+3)(x-1)(x+1)^{2}+\varepsilon f(x) y,
\end{aligned}
$$

with special elliptic Hamiltonian function:

$$
\tilde{H}(x, y)=\frac{1}{2} y^{2}+\frac{1}{6} x^{6}+\frac{2}{7} x^{5}-\frac{1}{7} x^{4}-\frac{10}{21} x^{3}-\frac{3}{14} x^{2},
$$

where $0<\varepsilon \ll 1, f(x)=\sum_{i=1}^{n} a_{i} x^{i}, a_{i}(i=0,1, \ldots, n)$ are real bounded parameters. System (6) has the degree $n^{*}=$ $\max \{5, n+1\}$. Let $H^{*}(5, n)$ denote the maximum number of limit cycles of (6) and $I^{*}\left(n^{*}\right)$ denote the maximum number of limit cycles of (6) of degree $n^{*}$. Our main interest is focused on $H^{*}(5,4)$.

The level sets (i.e., $\widetilde{H}(x, y)=h$ ) of Hamiltonian function (7) are sketched in Figure 1. $\widetilde{H}(x, y)=h$ defines the periodic orbits of system (6). There are a heteroclinic loop and a homoclinic loop for system $(6) \varepsilon=0$ defined by $\widetilde{H}(x, y)=0$, denoted by $L_{1}$ and $L_{2}$, respectively; let us in the following say $L^{*}=L_{1} \cup L_{2}$ is a hetero-homoclinic loop. $L_{1}$ connects the nilpotent cusp $S_{1}(-1,0)$ and the hyperbolic saddle $S_{2}(0,0)$ of system $(6) \varepsilon=0, L_{2}$ connects a the hyperbolic saddle $S_{2}(0,0)$ of system $(6) \varepsilon=0$. There are three families of clockwise periodic orbits, one family is $L_{1}^{h}=\{(x, y) \mid-25 / 2688<$ $\widetilde{H}(x, y)<0\}$ inside $L_{1}$ surrounding a center $C_{1}(-3 / 7,0)$ with $H(-3 / 7,0)=-25 / 2688$, one family is $L_{2}^{h}=\{(x, y) \mid-8 / 21<$ $\widetilde{H}(x, y)<0\}$ inside $L_{2}$ surrounding another center $C_{2}(1,0)$

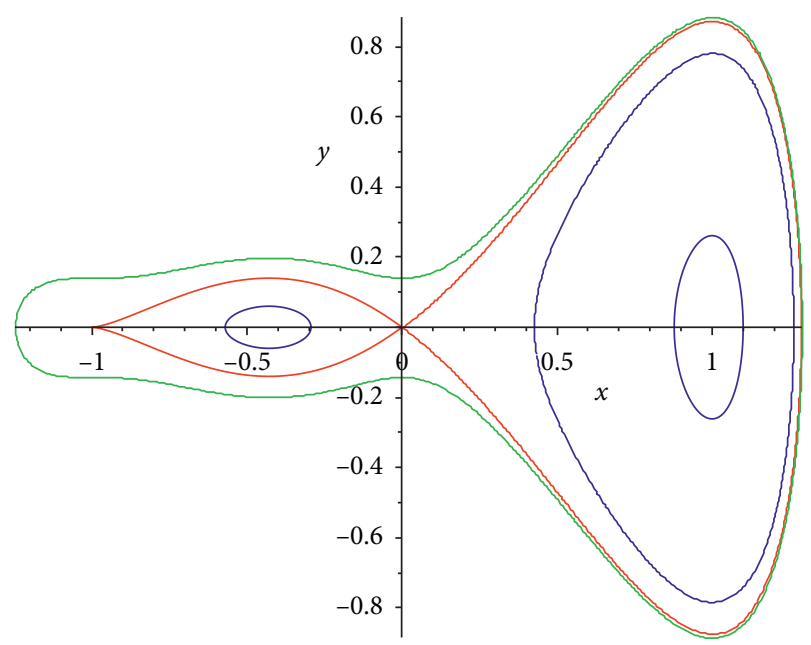

FIgURE 1: The portrait of system (6) when $\varepsilon=0$.

with $H(1,0)=-8 / 21$, and the third family is $L_{3}^{h}=$ $\{(x, y) \mid 0<\tilde{H}(x, y)<+\infty\}$ surrounding $L_{1}$ and $L_{2}$. Three families of periodic orbits form three periodic annuli, the boundaries of which are $L_{1}$ and $C_{1}, L_{2}$ and $C_{2}$, and $L^{*}$.

For system (6), we get the following main result.

Theorem 1. There exist some $a_{0}, a_{1}, a_{2}, a_{3}$ such that system (6) has 9 limit cycles for the type $(5,4)$ system (6). Therefore, $H(5,4) \geq H^{*}(5,4) \geq 9, I(5) \geq I^{*}(5) \geq 9$.

The rest of this paper is organized as follows. In Section 2 , we present some preliminaries which will be used in the next section. In Section 3, we study the asymptotic expansions of the related Melnikov functions for system (6). The proof of the main result is given in Section 4. Conclusion and discussions are drawn in Section 5.

\section{Preliminary Lemmas}

Consider the Melnikov function $M(h, \delta)$ for the nearHamiltonian system (1); we suppose the Hamiltonian system (2) has a bounded periodic annulus $\left\{\Gamma_{h}\right\}$ denoted by $\{H(x, y)=h\}$, and the period orbits in the periodic annulus are clockwise. The boundary of $\left\{\Gamma_{h}\right\}$ can be a center, a homoclinic loop, and a heteroclinic loop. We suppose the inner boundary of $\left\{\Gamma_{h}\right\}$ is defined by $H(x, y)=\alpha$ and the outer boundary is defined by $H(x, y)=\beta$; correspondingly, we have

$$
M(h, \delta)=\oint_{\Gamma_{h}} q \mathrm{~d} x-p \mathrm{~d} y, \quad \alpha<h<\beta,
$$

and $M(h, \delta)$ can be expanded near its boundary (see [20-23]).

When the inner boundary of $\left\{\Gamma_{h}\right\}$ is elementary center, we suppose it is located at $C\left(x_{c}, y_{c}\right)$ with $H\left(x_{c}, y_{c}\right)=\alpha$, and for $(x, y)$ near $C\left(x_{c}, y_{c}\right)$, 


$$
\begin{aligned}
H(x, y)= & \alpha+\frac{1}{2}\left(x-x_{c}\right)^{2}+\frac{1}{2}\left(y-y_{c}\right)^{2} \\
& +\sum_{i+j \geq 3} \widetilde{h}_{i j}\left(x-x_{c}\right)^{i}\left(y-y_{c}\right)^{j}, \\
p(x, y, \delta)= & \sum_{i+j \geq 0} \tilde{a}_{i j}\left(x-x_{c}\right)^{i}\left(y-y_{c}\right)^{j}, \\
q(x, y, \delta)= & \sum_{i+j \geq 0} \widetilde{b}_{i j}\left(x-x_{c}\right)^{i}\left(y-y_{c}\right)^{j} .
\end{aligned}
$$

For the expansion of $M(h, \delta)$ near $C\left(x_{c}, y_{c}\right)$, we have the following.

Lemma 1 (see [20]). Under the condition we suppose above, for the expansion of $M(h, \delta)$ near the elementary center $C\left(x_{c}, y_{c}\right)(h=\alpha)$, we have

$$
M(h, \delta)=\sum_{j \geq 0} b_{j}(\delta)(h-\alpha)^{j+1}, \quad \alpha<h \ll 1 .
$$

Under (9), the formulas of $b_{j}$ can be obtained by using the programs in [20].

When the outer boundary of $\left\{\Gamma_{h}\right\}$ is homoclinic loop denoted by $\Gamma_{\beta}$ passing through a hyperbolic saddle $S\left(x_{s}, y_{s}\right)$ satisfying $H\left(x_{s}, y_{s}\right)=\beta$, for the expansion of $M(h, \delta)$ near the homoclinic loop, we have the following.

\section{Lemma 2.}

(i) (see [21]). Under the condition we suppose above, the function $M(h, \delta)$ has the following expansion:

$$
\begin{aligned}
M(h, \delta)= & c_{0}(\delta)+c_{1}(\delta)(h-\beta) \ln |h-\beta|+c_{2}(\delta)(h-\beta) \\
& +c_{3}(\delta)(h-\beta)^{2} \ln |h-\beta|+O\left(|h-\beta|^{2}\right),
\end{aligned}
$$

for $0<-(h-\beta) \ll 1$, where $c_{1}(\delta)$ and $c_{3}(\delta)$ depend on the coefficients of $H(x, y), p(x, y)$, and $q(x, y)$.

$$
\begin{aligned}
& c_{0}(\delta)=M(\beta, \delta)=\left.\oint_{\Gamma_{\beta}}(q \mathrm{~d} x-p \mathrm{~d} y)\right|_{\varepsilon=0}, \\
& c_{2}(\delta)=\oint_{\Gamma_{\beta}}\left(p_{x}+q_{y}-\sigma\right) \mathrm{d} t+b c_{1}(\delta),
\end{aligned}
$$

for $\sigma=\left.\left(p_{x}+q_{y}\right)\right|_{x=x_{s}, y=y_{s}}$ is divergence at $S\left(x_{s}, y_{s}\right)$, $b$ is a constant.

(ii) (see [21]). In particular, for $(x, y)$ near the hyperbolic saddle $S\left(x_{s}, y_{s}\right)$,

$$
\begin{aligned}
H(x, y)= & \beta+\frac{\lambda}{2}\left(y-y_{s}\right)^{2}-\frac{1}{2}\left(x-x_{s}\right)^{2} \\
& +\sum_{i+j \geq 2} \bar{h}_{i j}\left(x-x_{s}\right)^{i}\left(y-y_{s}\right)^{j}, \\
p(x, y, \delta)= & \sum_{i+j \geq 0} \bar{a}_{i j}\left(x-x_{s}\right)^{i}\left(y-y_{s}\right)^{j}, \\
q(x, y, \delta)= & \sum_{i+j \geq 0} \bar{b}_{i j}\left(x-x_{s}\right)^{i}\left(y-y_{s}\right)^{j} .
\end{aligned}
$$

Then,

$$
\begin{aligned}
c_{1}(\delta)= & -\frac{1}{|\lambda|}\left(\bar{a}_{10}+\bar{b}_{01}\right), \\
c_{3}(\delta)= & \frac{-1}{2|\lambda| \lambda}\left\{\left(-3 \bar{a}_{30}-\bar{b}_{21}+\bar{a}_{12}+3 \bar{b}_{03}\right)\right. \\
& -\frac{1}{\lambda}\left[\left(2 \bar{b}_{02}+\bar{a}_{11}\right)\left(3 \bar{h}_{03}-\bar{h}_{21}\right)\right. \\
& \left.\left.+\left(2 \bar{a}_{20}+\bar{b}_{11}\right)\left(\bar{h}_{30}-\bar{h}_{12}\right)\right]\right\} .
\end{aligned}
$$

Definition 1 . The values $c_{1}(\delta)$ and $c_{3}(\delta)$ are, respectively, called the first and second local Melnikov coefficients at the saddle $S\left(x_{s}, y_{s}\right)$, denoted by $c_{1}\left(S_{\text {saddle }}, \delta\right), \quad c_{3}\left(S_{\text {saddle }}, \delta\right)$, respectively.

Note.

(i) The local coefficients are presented in the expansion of a Melnikov function, which are local quantities at a singular point depending on the coefficients of the expansions of $H(x, y), p(x, y)$, and $q(x, y)$ at the singular point.

(ii) When there is a nilpotent cusp $S\left(x_{n}, y_{n}\right)$ connecting a homoclinic loop, there are similarly some local coefficients at the cusp $S\left(x_{n}, y_{n}\right)$ presented in the expansion of the corresponding Melnikov function near the homoclinic loop; we denote these first three local coefficients by $c_{1}\left(S_{\text {cusp }}, \delta\right), c_{3}\left(S_{\text {cusp }}, \delta\right)$, and $c_{4}\left(S_{\text {cusp }}, \delta\right)($ see $[22])$.

Lemma 3 (see [22]). Suppose there is a nilpotent cusp $S\left(x_{n}, y_{n}\right)$ which is a limit point of a homoclinic loop or a heteroclinic loop, and for $(x, y)$ near the nilpotent cusp $S\left(x_{n}, y_{n}\right)$ :

$$
\begin{aligned}
H(x, y)= & \beta+\frac{1}{2}\left(y-y_{n}\right)^{2} \\
& +\sum_{i+j \geq 3} h_{i j}\left(x-x_{n}\right)^{i}\left(y-y_{n}\right)^{j}, \quad h_{30}<0, \\
p(x, y, \delta)= & \sum_{i+j \geq 0} a_{i j}\left(x-x_{n}\right)^{i}\left(y-y_{n}\right)^{j}, \\
q(x, y, \delta)= & \sum_{i+j \geq 0} b_{i j}\left(x-x_{n}\right)^{i}\left(y-y_{n}\right)^{j} .
\end{aligned}
$$

Then, the first three local coefficients of the corresponding Melnikov functions at the nilpotent cusp $S\left(x_{n}, y_{n}\right)$ are as follows: 


$$
\begin{aligned}
& c_{1}\left(S_{\text {cusp }}, \delta\right)=2 \sqrt{2} h_{30}^{-1 / 3}\left[a_{10}+b_{01}\right] \text {, } \\
& c_{3}\left(S_{\text {cusp }}, \delta\right)=2 \sqrt{2} h_{30}^{-5 / 3}\left[h_{30}\left(2 a_{20}+b_{11}-h_{12}\left(a_{10}+b_{01}\right)\right)+1 / 3\left(h_{21}^{2}-2 h_{40}\right)\left(a_{10}+b_{01}\right)\right], \\
& c_{4}\left(S_{\text {cusp }}, \delta\right)=9 \mu_{1}^{-1} \alpha_{01}-2 \mu_{1}^{-7}\left[\left(20 \mu_{2}^{3}-20 \mu_{1} \mu_{2} \mu_{3}+4 \mu_{1}^{2} \mu_{4}\right) \alpha_{00}+\left(4 \mu_{1}^{2} \mu_{3}-10 \mu_{1} \mu_{2}^{2}\right) \alpha_{10}+4 \mu_{1}^{2} \mu_{2} \alpha_{20}-\mu_{1}^{3} \alpha_{30}\right] \text {, } \\
& \mu_{1}=\sqrt[3]{h_{30}} \\
& \mu_{2}=-\frac{1}{6} h_{30}^{-2 / 3}\left(-2 h_{40}+h_{21}^{2}\right) \text {, } \\
& \mu_{3}=\frac{1}{36} h_{30}^{-5 / 3}\left[12 h_{30}\left(h_{50}-h_{31} h_{21}+h_{12} h_{21}^{2}\right)-4 h_{40}^{2}+4 h_{40} h_{21}^{2}-h_{21}^{4}\right] \text {, } \\
& \mu_{4}=-\frac{1}{648} h_{30}^{-8 / 3}\left[\begin{array}{c}
5 h_{21}^{6}-40 h_{40}^{3}+30 h_{40} h_{21}^{2}\left(2 h_{40}-h_{21}^{2}\right)+144 h_{30} h_{40}\left(h_{50}-h_{31} h_{21}+h_{12} h_{21}^{2}\right) \\
+72 h_{30} h_{21}^{2}\left(-h_{50}+h_{31} h_{21}-h_{12} h_{21}^{2}\right)+216 h_{30}^{2}\left(-h_{60}-h_{22} h_{21}^{2}+h_{41} h_{21}+h_{03} h_{21}^{3}\right) \\
+108 h_{30}^{2} h_{31}^{2}+432 h_{30}^{2} h_{12} h_{21}\left(h_{12} h_{21}-h_{31}\right)
\end{array}\right], \\
& \alpha_{00}=2 \sqrt{2}\left(a_{10}+b_{01}\right) \\
& \alpha_{10}=2 \sqrt{2}\left[-h_{12}\left(a_{10}+b_{01}\right)+2 a_{20}+b_{11}\right] \text {, } \\
& \alpha_{20}=2 \sqrt{2}\left[\left(a_{10}+b_{01}\right)\left(3 h_{03} h_{21}-h_{22}+\frac{3}{2} h_{12}^{2}\right)-2 h_{12} a_{20}-h_{12} b_{11}+3 a_{30}+b_{21}-a_{11} h_{21}-2 b_{02} h_{21}\right], \\
& \alpha_{30}=2 \sqrt{2}\left[\begin{array}{c}
\left(a_{10}+b_{01}\right)\left(3 h_{13} h_{21}+3 h_{03} h_{31}+3 h_{12} h_{22}-15 h_{12} h_{03} h_{21}-\frac{5}{2} h_{12}^{3}-h_{32}\right)+\left(3 a_{11}+6 b_{02}\right) \\
h_{12} h_{21}-2\left(b_{12}+a_{21}\right) h_{21}-\left(a_{11}+2 b_{02}\right) h_{31}+4 a_{40}+b_{31}+\left(3 b_{11}+6 a_{20}\right) \\
h_{03} h_{21}-\left(2 a_{20}+b_{11}\right) h_{22}+\left(3 a_{20}+\frac{3}{2} b_{11}\right) h_{12}^{2}-\left(3 a_{30}+b_{21}\right) h_{12}
\end{array}\right], \\
& \alpha_{01}=2 \sqrt{2}\left[\frac{2}{3} a_{12}+2 b_{03}-2 h_{03} a_{11}-4 h_{03} b_{02}+\left(a_{10}+b_{01}\right)\left(5 h_{03}^{2}-2 h_{04}\right)\right] \text {. }
\end{aligned}
$$

When the outer boundary of $\Gamma_{h}$ is heteroclinic loop denoted by $\Gamma_{\beta}$ passing through a hyperbolic saddle $S\left(x_{s}, y_{s}\right)$ and a nilpotent cusp $N\left(x_{n}, y_{n}\right)$ satisfying $H\left(x_{s}, y_{s}\right)=H\left(x_{n}, y_{n}\right)=\beta$, for the expansion of $M(h, \delta)$ near the heteroclinic loop $\Gamma_{\beta}$, we have the following.

Lemma 4 (see [23]). The expansion of $M(h, \delta)$ near the heteroclinic loop $L_{\beta}$ has the form

$$
\begin{aligned}
M(h, \delta)= & \widetilde{c}_{0}(\delta)+B_{00} \widetilde{c}_{1}(\delta)|h-\beta|^{5 / 6}+\widetilde{c}_{2}(\delta)(h-\beta) \ln |h-\beta| \\
& +\left[\widetilde{c}_{3}(\delta)+b_{1} \widetilde{c}_{1}(\delta)+b_{2} \widetilde{c}_{2}(\delta)\right] \\
& \cdot(h-\beta)+B_{10} \widetilde{c}_{4}(\delta)|h-\beta|^{7 / 6} \\
& +\widetilde{c}_{5}(\delta)(h-\beta)^{2} \ln |h-\beta| \\
& -\frac{1}{11} B_{00} \widetilde{c}_{6}(\delta)|h-\beta|^{11 / 6}+O\left((h-\beta)^{2}\right) .
\end{aligned}
$$

for $0<-(h-\beta) \ll 1$, where $B_{00}=3 / 5 \int_{0}^{1} d v / \sqrt{v\left(1-v^{3}\right)}=$ $3 / 5 \times 2.4286 \ldots$,
$B_{10}=-3 / 7 \int_{0}^{1}\left(v^{(3 / 2) d v} / \sqrt{1-v^{3}}\left(1+\sqrt{1-v^{3}}\right)-2\right)>0, \quad b_{1}$ and $b_{2}$ are constants, and

$$
\begin{aligned}
& \widetilde{c}_{0}(\delta)=M(\beta, \delta) \\
& \tilde{c}_{1}(\delta)=c_{1}\left(N_{\text {cusp }}, \delta\right), \\
& \widetilde{c}_{2}(\delta)=c_{1}\left(S_{\text {saddle }}, \delta\right), \\
& \widetilde{c}_{4}(\delta)=c_{3}\left(N_{\text {cusp }}, \delta\right), \\
& \widetilde{c}_{5}(\delta)=c_{3}\left(S_{\text {saddle }}, \delta\right), \\
& \widetilde{c}_{6}(\delta)=c_{4}\left(N_{\text {cusp }}, \delta\right),
\end{aligned}
$$

and $\widetilde{c}_{3}(\delta) \in R$. In particular, if $\widetilde{c}_{1}(\delta)=\widetilde{c}_{2}(\delta)$, we have

$$
\widetilde{c}_{3}(\delta)=\oint_{\Gamma_{\beta}}\left(p_{x}+q_{y}\right) \mathrm{d} t .
$$

In many cases, the Hamiltonian function is not of the form supposed in the above lemmas. Then, to apply the lemmas, we need to first introduce suitable linear change of variables which will cause a change of the first-order Melnikov function. The following lemma gives the relationship between the old and new Melnikov functions. 
Lemma 5 (see [2]). Under the linear change of variables of the form

$$
\begin{aligned}
& u=a\left(x-x_{0}\right)+b\left(y-y_{0}\right), \\
& v=c\left(x-x_{0}\right)+d\left(y-y_{0}\right),
\end{aligned}
$$

and time rescaling $\tau=k t$, where $D=a d-b c \neq 0$, system (31) becomes

$$
\begin{aligned}
& \frac{\mathrm{d} u}{\mathrm{~d} \tau}=\widetilde{H}_{v}+\varepsilon \tilde{p}, \\
& \frac{\mathrm{d} v}{\mathrm{~d} \tau}=-\widetilde{H}_{u}+\varepsilon \widetilde{q},
\end{aligned}
$$

where

$$
\begin{aligned}
\widetilde{H}(u, v) & =\frac{D}{k} H(x, y), \\
\widetilde{p}(u, v, \delta) & =\frac{1}{k}[a p(x, y, \delta)+b q(x, y, \delta)], \\
\widetilde{q}(u, v, \delta) & =\frac{1}{k}[c p(x, y, \delta)+d q(x, y, \delta)] .
\end{aligned}
$$

Let

$$
\widetilde{M}(h, \delta)=\left.\oint_{\widetilde{\Gamma}_{h}}(\widetilde{q} \mathrm{~d} u-\widetilde{p} \mathrm{~d} v)\right|_{\varepsilon=0},
$$

which is the Melnikov function of system (21). Then,

$$
M(h, \delta)=\frac{|k|}{D} \widetilde{M}\left(\frac{D}{k} h, \delta\right) .
$$

Remark 1. Usually when we apply Lemma 5, we always take a linear transformation such that $D / k=1$ because under this condition, the local coefficients between the old and the new ones, respectively, are the same (if $|k| / D=1$ ) or only different from a symbol "-" (if $|k| / D=-1$ ).

Let us now suppose system (2) has a hetero-homoclinic loop $\Gamma^{*}=\Gamma_{1} \cup \Gamma_{2}$, where $\Gamma_{1}$ is heteroclinic loop connecting a cusp $S_{1}\left(x_{1}, y_{1}\right)$ and a hyperbolic saddle $S_{2}\left(x_{2}, y_{2}\right)$ satisfying $H\left(x_{1}, y_{1}\right)=H\left(x_{2}, y_{2}\right)=\beta$ and $\Gamma_{2}$ is homoclinic loop passing through the previous hyperbolic saddle $S\left(x_{2}, y_{2}\right)$. There exist three families of orbits, the first family of periodic orbits $\Gamma_{h}^{1}$ surrounding a center $C_{1}\left(x_{3}, y_{3}\right)$ with $H\left(x_{3}, y_{3}\right)=$ $\alpha_{1}$ in $\Gamma_{1}$, the second family of periodic orbits $\Gamma_{h}^{2}$ surrounding another center $C_{2}\left(x_{4}, y_{4}\right)$ with $H\left(x_{4}, y_{4}\right)=\alpha_{2}$ in $\Gamma_{2}$, and the third family of periodic orbits $\Gamma_{h}^{*}$ surrounding $\Gamma^{*}$. The portrait of $\Gamma^{*}$ is just the same as Figure 1.

Correspondingly, we have three Melnikov functions:

$$
\begin{aligned}
& M_{i}(h, \delta)=\oint_{\Gamma_{h}^{i}} q \mathrm{~d} x-p \mathrm{~d} y, \quad \text { for } \alpha_{i}<h<\beta, i=1,2, \\
& M_{3}(h, \delta)=\oint_{\Gamma_{h}^{3}} q \mathrm{~d} x-p \mathrm{~d} y, \quad \text { for } \beta<h<+\infty .
\end{aligned}
$$

From Lemmas 2-4, we have the following.
Lemma 6. Under the condition supposed above, the three Melnikov functions have the following expansions:

$$
\begin{aligned}
M_{1}(h, \delta)= & e_{0}(\delta)+B_{00} e_{1}(\delta)|h-\beta|^{5 / 6}+e_{2}(\delta)(h-\beta) \ln |h-\beta| \\
& +\left[e_{3}(\delta)+b_{1} e_{1}(\delta)+b_{2} e_{2}(\delta)\right](h-\beta) \\
& +B_{10} e_{4}(\delta)|h-\beta|^{7 / 6}+e_{5}(\delta)(h-\beta)^{2} \\
& \cdot \ln |h-\beta|-\frac{1}{11} B_{00} e_{6}(\delta)|h-\beta|^{11 / 6}+O\left((h-\beta)^{2}\right),
\end{aligned}
$$

for $0<-(h-\beta) \ll 1$.

$$
\begin{aligned}
M_{2}(h, \delta)= & c_{0}(\delta)+c_{1}(\delta)(h-\beta) \ln |h-\beta|+c_{2}(\delta)(h-\beta) \\
& +c_{3}(\delta)(h-\beta)^{2} \ln |h-\beta|+O\left(|h-\beta|^{2}\right),
\end{aligned}
$$

$$
\begin{aligned}
& \text { for } 0<-(h-\beta) \ll 1 . \\
& \qquad \begin{aligned}
M_{3}(h, \delta)= & \widetilde{c}_{0}(\delta)+B_{00}^{*} \widetilde{c}_{1}(\delta)|h-\beta|^{5 / 6}+\widetilde{c}_{2}(\delta)(h-\beta) \\
& \cdot \ln |h-\beta|+\left[\widetilde{c}_{3}(\delta)+b_{3} \widetilde{c}_{1}(\delta)+b_{4} \widetilde{c}_{2}(\delta)\right](h-\beta) \\
& +B_{10}^{*} \widetilde{c}_{4}(\delta)|h-\beta|^{7 / 6}+\widetilde{c}_{5}(\delta)(h-\beta)^{2} \ln |h-\beta| \\
& -\frac{1}{11} B_{00}^{*} \widetilde{c}_{6}(\delta)|h-\beta|^{11 / 6}+O\left((h-\beta)^{2}\right),
\end{aligned}
\end{aligned}
$$

for $0<(h-\beta) \ll 1$, where $B_{00}$ and $B_{10}$ are the same as before, $B_{00}^{*}<0$ and $B_{10}^{*}<0, b_{1}, b_{2}, b_{3}$ and $b_{4}$ are some constants. and $e_{1}(\delta)=c_{1}\left(S_{1_{\text {cusp }}}, \delta\right), \quad e_{2}(\delta)=c_{1}\left(S_{2_{\text {saddle }}}, \delta\right), \quad e_{4}(\delta)=c_{3}\left(S_{1_{\text {cusp }},}\right.$, $\delta), e_{5}(\delta)=c_{3}\left(S_{2_{\text {saddle }}} \delta\right), e_{6}(\delta)=c_{4}\left(S_{1_{\text {cuss }}}, \delta\right), c_{1}(\delta)=c_{1}\left(S_{2_{\text {saddlle }}}\right.$, $\delta), \quad c_{3}(\delta)=c_{3}\left(S_{2_{\text {saddle }}}, \delta\right), \quad \widetilde{c}_{1}(\delta)=c_{1}\left(S_{1_{\text {cusp }}}, \delta\right), \quad \tilde{c}_{2}(\delta)=2 c_{1}$ $\left(S_{2_{\text {saddle }}}, \delta\right), \tilde{c}_{4}(\delta)=c_{3}\left(S_{1_{\text {cusp }}}, \delta\right), \tilde{c}_{5}(\delta)=2 c_{3}\left(S_{2_{\text {saddle }}}, \delta\right), \tilde{c}_{6}(\delta)=$ $c_{4}\left(S_{1_{\text {cusp }}}, \delta\right)$, where $c_{1}\left(S_{1_{\text {cusp }}}, \delta\right), c_{3}\left(S_{1_{\text {cusp }}}, \delta\right)$, and $c_{4}\left(S_{1_{\text {cusp }}}, \delta\right)$ are local coefficients at the nilpotent cusp $S_{1}\left(x_{1}, y_{1}\right)$ which can be obtained by Lemma 3 and $c_{1}\left(S_{2_{\text {saddle }}}, \delta\right)$ and $c_{3}\left(S_{2_{\text {saddle }}}, \delta\right)$ are local coefficients at the hyperbolic saddle $S_{2}\left(x_{2}, y_{2}\right)$ which can be obtained by Lemma 2, while

$$
\begin{aligned}
e_{0}(\delta) & =M_{1}(\beta, \delta)=\oint_{\Gamma_{1}} q \mathrm{~d} x-p \mathrm{~d} y, c_{0}(\delta)=M_{2}(\beta, \delta) \\
& =\oint_{\Gamma_{2}} q \mathrm{~d} x-p \mathrm{~d} y, \widetilde{c}_{0}(\delta)=e_{0}+c_{0},
\end{aligned}
$$

and $e_{3}(\delta) \in R$; in particular, if $c_{1}\left(S_{2_{\text {saddle }}}, \delta\right)=c_{1}\left(S_{1_{\text {cusp }}}, \delta\right)=0$, we have

$$
e_{3}(\delta)=\oint_{\Gamma_{1}}\left(p_{x}+q_{y}\right) \mathrm{d} t
$$

and $c_{2}(\delta)=\left.\oint_{\Gamma_{2}}\left(p_{x}+q_{y}-a_{10}-b_{01}\right)\right|_{\varepsilon=0} d t+b c_{1}(\delta) \in R$; in particular, if $c_{1}\left(S_{2_{\text {saddle }}}, \delta\right)=0$, we have 


$$
c_{2}(\delta)=\oint_{\Gamma_{2}}\left(p_{x}+q_{y}\right) \mathrm{d} t
$$

and $\widetilde{c}_{3}(\delta)=\oint_{\Gamma_{1}}\left(p_{x}+q_{y}\right) d t+\oint_{\Gamma_{2}}\left(p_{x}+q_{y}\right) d t(\delta)$ if $c_{1}\left(S_{2_{\text {saddle }}}\right.$, $\delta)=c_{1}\left(S_{1_{\text {cus }}}, \delta\right)=0$.

\section{The Expansions of Melnikov Functions of System (6)}

Liénard system (6) is a special form of near-Hamiltonian system (1) with $H(x, y)=\widetilde{H}(x, y), p(x, y)=0, q(x, y)=$ $f(x) y=\sum_{i=0}^{4} a_{i} x^{i} y$. In this section, we take $n=4$; then, system $(6)$ is a Liénard system of type $(5,4)$. There are three Melnikov functions corresponding to three periodic annuli of system $(6) \varepsilon=0$

$$
\begin{array}{ll}
M_{1}(h, \delta)=\oint_{L_{1}^{h}} f(x) y \mathrm{~d} x, & \text { for }-\frac{25}{2688}<h<0, \\
M_{2}(h, \delta)=\oint_{L_{2}^{h}} f(x) y \mathrm{~d} x, & \text { for }-\frac{8}{21}<h<0, \\
M_{3}(h, \delta)=\oint_{L_{3}^{h}} f(x) y \mathrm{~d} x, & \text { for } 0<h<+\infty .
\end{array}
$$

By Theorem 1, we obtain

$$
\begin{aligned}
M_{1}(h, \delta)= & e_{0}(\delta)+B_{00} e_{1}(\delta)|h|^{5 / 6}+e_{2}(\delta) h \ln |h| \\
& +\left[e_{3}(\delta)+b_{1} e_{1}(\delta)+b_{2} e_{2}(\delta)\right](h) \\
& +B_{10} e_{4}(\delta)|h|^{7 / 6}+e_{5}(\delta) h^{2} \ln |h| \\
& -\frac{1}{11} B_{00} e_{6}(\delta)|h|^{11 / 6}+O\left(h^{2}\right),
\end{aligned}
$$

for $0<-h \ll 1$.

$$
\begin{aligned}
M_{2}(h, \delta)= & c_{0}(\delta)+c_{1}(\delta) h \ln |h|+c_{2}(\delta) h \\
& +c_{3}(\delta) h^{2} \ln |h|+O\left(h^{2}\right),
\end{aligned}
$$

for $0<-h \ll 1$.

$$
\begin{aligned}
M_{3}(h, \delta)= & \widetilde{c}_{0}(\delta)+B_{00}^{*} \widetilde{c}_{1}(\delta) h^{5 / 6}+\widetilde{c}_{2}(\delta) h \ln h \\
& +\left[\widetilde{c}_{3}(\delta)+b_{3} \widetilde{c}_{1}(\delta)+b_{4} \widetilde{c}_{2}(\delta)\right] h \\
& +B_{10}^{*} \widetilde{c}_{4}(\delta) h^{7 / 6}+\widetilde{c}_{5}(\delta) h^{2} \ln h \\
& +\frac{1}{11} B_{00}^{*} \widetilde{c}_{6}(\delta) h^{11 / 6}+O\left(h^{2}\right),
\end{aligned}
$$

for $0<h \ll 1$.

By Lemma 1 , for $0<h+25 / 2688 \ll 1$,

$$
M_{1}(h, \delta)=\sum_{i=0} b_{i}(\delta)\left(h+\frac{25}{2688}\right)^{i+1}
$$

and for $0<h+8 / 21 \ll 1$,

$$
M_{2}(h, \delta)=\sum_{i=0} d_{i}(\delta)\left(h+\frac{8}{21}\right)^{i+1} .
$$

In the following, we use the preliminary lemmas to compute the coefficients of the above expansions for $M_{1}(h, \delta), M_{2}(h, \delta)$, and $M_{3}(h, \delta)$. Firstly, we have

$$
\begin{aligned}
e_{0}(\delta) & =M_{1}(0, \delta)=\oint_{L_{1}} f(x) y \mathrm{~d} x \\
& =2 \int_{-1}^{0} f(x) \sqrt{-2 A(x)} \mathrm{d} x=\sum_{i=0}^{9} k_{i} a_{i},
\end{aligned}
$$

where

$$
\begin{aligned}
& k_{0}=\frac{741 \sqrt{21}}{4802}-\frac{256 \sqrt{3}}{2401} \pi+\frac{512 \sqrt{3}}{2401} \arcsin \left(\frac{1}{8}\right), \\
& k_{1}=\frac{1191 \sqrt{21}}{12005}-\frac{256 \sqrt{3}}{2401} \pi+\frac{512 \sqrt{3}}{2401} \arcsin \left(\frac{1}{8}\right), \\
& k_{2}=\frac{17467 \sqrt{21}}{168070}-\frac{4352 \sqrt{3}}{50421} \pi+\frac{8704 \sqrt{3}}{50421} \arcsin \left(\frac{1}{8}\right), \\
& k_{3}=\frac{382302 \sqrt{21}}{4117715}-\frac{74496 \sqrt{3}}{823543} \pi+\frac{148992 \sqrt{3}}{823543} \arcsin \left(\frac{1}{8}\right), \\
& k_{4}=\frac{11525967 \sqrt{21}}{115296020}-\frac{506112 \sqrt{3}}{5764801} \pi+\frac{1012224 \sqrt{3}}{5764801} \arcsin \left(\frac{1}{8}\right) .
\end{aligned}
$$

Secondly,

$$
\begin{aligned}
c_{0}(\delta) & =M_{2}(0, \delta)=\oint_{L_{2}} f(x) y \mathrm{~d} x \\
& =2 \int_{0}^{9 / 7} f(x) \sqrt{-2 A(x)} \mathrm{d} x=\sum_{i=0}^{9} I_{i} a_{i},
\end{aligned}
$$

where

$$
\begin{aligned}
& I_{0}=\frac{741 \sqrt{21}}{4802}+\frac{256 \sqrt{3}}{2401} \pi+\frac{512 \sqrt{3}}{2401} \arcsin \left(\frac{1}{8}\right), \\
& I_{1}=\frac{1191 \sqrt{21}}{12005}+\frac{256 \sqrt{3}}{2401} \pi+\frac{512 \sqrt{3}}{2401} \arcsin \left(\frac{1}{8}\right), \\
& I_{2}=\frac{17467 \sqrt{21}}{168070}+\frac{4352 \sqrt{3}}{50421} \pi+\frac{8704 \sqrt{3}}{50421} \arcsin \left(\frac{1}{8}\right), \\
& I_{3}=\frac{382302 \sqrt{21}}{4117715}+\frac{74496 \sqrt{3}}{823543} \pi+\frac{148992 \sqrt{3}}{823543} \arcsin \left(\frac{1}{8}\right), \\
& I_{4}=\frac{11525967 \sqrt{21}}{115296020}+\frac{506112 \sqrt{3}}{5764801} \pi+\frac{1012224 \sqrt{3}}{5764801} \arcsin \left(\frac{1}{8}\right) .
\end{aligned}
$$

Therefore,

$$
\widetilde{c}_{0}(\delta)=e_{0}(\delta)+c_{0}(\delta)=\sum_{i=1}^{9} \widetilde{I}_{i} a_{i},
$$


where $\widetilde{I}_{i}=k_{i}+I_{i}$.

In order to get the local coefficients $c_{1}\left(S_{1_{\text {cusp }}}, \delta\right)$ at the cusp $S_{1}(-1,0)$, we introduce the transformation $u:=x+1, v:=y$; then, system (6) becomes

$$
\begin{aligned}
& \dot{u}=v, \\
& \dot{v}=-u^{5}+\frac{25}{7} u^{4}-\frac{26}{7} u^{3}+\frac{8}{7} u^{2}+\varepsilon \bar{q}(u, v),
\end{aligned}
$$

with Hamiltonian function $\bar{H}(u, v)=1 / 6 u^{6}-5 / 7 u^{5}+13 /$ $14 u^{4}-8 / 21 u^{3}+1 / 2 v^{2}$, $\bar{q}(u, v)=\left.f(x) y\right|_{x=u-1, y=v},\left.\bar{q}_{v}\right|_{u=0, v=0}=a_{0}-a_{1}+a_{2}-a_{3}+a_{4}$.

Applying the formula for $c_{1}\left(S_{1_{\text {cusp }}}, \delta\right), c_{3}\left(S_{1_{\text {cusp }}}, \delta\right)$, $c_{4}\left(S_{1_{\text {cusp }}}, \delta\right)$ in Lemma 3 , in accordance with Lemma 6 , we have

$$
\begin{aligned}
e_{1}(\delta)= & \widetilde{c}_{1}(\delta)=c_{1}\left(S_{1_{\text {cusp }}}, \delta\right) \\
= & -21^{1 / 3} \sqrt{2}\left(a_{0}-a_{1}+a_{2}-a_{3}+a_{4}\right), \\
e_{4}(\delta)= & \widetilde{c}_{4}(\delta)=c_{3}\left(S_{1_{\text {cusp }}}, \delta\right) \\
= & -\frac{21^{2 / 3} \sqrt{2}}{16}\left(13 a_{0}-5 a_{1}-3 a_{2}+11 a_{3}-19 a_{4}\right), \\
e_{6}(\delta)= & \widetilde{c}_{6}(\delta)=c_{4}\left(S_{1_{\text {cusp }}}, \delta\right) \\
= & \frac{21 \sqrt[3]{21} \sqrt{2}}{4096} \\
& \cdot\left(11669 a_{0}-4765 a_{1}+1189 a_{2}+83 a_{3}-75 a_{4}\right) .
\end{aligned}
$$

In order to get the local coefficient $c_{1}\left(S_{2_{\text {saddle }}}, \delta\right)$ near the hyperbolic saddle $S_{2}(0,0)$, we introduce the transformation $u:=\sqrt{3 / 7} x, v:=y, \tau=\sqrt{3 / 7} t$; then, system (6) becomes

$$
\begin{aligned}
& \frac{\mathrm{d} u}{\mathrm{~d} \tau}=v, \\
& \frac{\mathrm{d} v}{\mathrm{~d} \tau}=-\frac{343}{27} u^{5}-\frac{70}{27} \sqrt{21} u^{4}+\frac{28}{9} u^{3}+\frac{10}{9} \sqrt{21} u^{2}+u
\end{aligned}
$$

with Hamiltonian system $\hat{H}(u, v)=343 / 162 u^{6}+14 / 27$ $\sqrt{21} u^{5}-7 / 9 u^{4}-10 / 27 \sqrt{21} u^{3} 1 / 2 u^{2}+1 / 2 v^{2}$, and $\hat{q}(u, v)=$ $\left.\sqrt{3 / 7}\left(\sum_{i=0} a_{i} x^{i} y\right)\right|_{x=\sqrt{7 / 3}, y=v}$. Applying the formula for $c_{1}\left(S_{2_{\text {saddle }}}, \delta\right)$ and $c_{3}\left(S_{2_{\text {saddle }}}, \delta\right)$ in Lemma 2 , in accordance with Lemma 5, we have

$$
\begin{aligned}
& e_{2}(\delta)=c_{1}(\delta)=\widetilde{c}_{2}(\delta)=c_{1}\left(S_{2_{\text {saddle }}}, \delta\right)=-\frac{1}{3} \sqrt{21} a_{0}, \\
& \tilde{c}_{2}(\delta)=2 c_{1}\left(S_{2_{\text {saddle }}}, \delta\right)=-\frac{2}{3} \sqrt{21} a_{0}, \\
& e_{5}(\delta)=c_{3}(\delta)=c_{3}\left(S_{2_{\text {saddle }}}, \delta\right)=-\frac{7 \sqrt{21}}{54}\left(10 a_{1}-3 a_{2}\right), \\
& \widetilde{c}_{5}(\delta)=2 c_{3}\left(S_{2_{\text {saddle }}}, \delta\right)=-\frac{7 \sqrt{21}}{27}\left(10 a_{1}-3 a_{2}\right) .
\end{aligned}
$$

Let $e_{1}(\delta)=e_{2}(\delta)=0$; we have

$$
\begin{aligned}
& a_{0}=0, \\
& a_{1}=a_{2}-a_{3}+a_{4} .
\end{aligned}
$$

Under this case,

$$
\begin{aligned}
e_{3}(\delta) & =\oint_{L_{1}} f(x) \mathrm{d} t=\oint_{L_{1}} \frac{f(x)}{y} \mathrm{~d} x=\oint_{L_{1}} \frac{f(x)}{\sqrt{-2 A(x)}} \mathrm{d} x \\
& =2 \int_{-1}^{0} \frac{f(x)}{\sqrt{-2 A(x)}} \mathrm{d} x .
\end{aligned}
$$

Taking (47) into (48), we have with the help of Maple 13,

$$
e_{3}(\delta)=\sum_{i=2}^{9} l_{i} a_{i},
$$

where

$$
\begin{aligned}
& l_{2}=2 \sqrt{3} \arcsin \left(\frac{1}{8}\right)-\sqrt{3} \pi, \\
& l_{3}=-\frac{12}{7} \sqrt{3} \arcsin \left(\frac{1}{8}\right)+\frac{6}{7} \sqrt{3} \pi+\frac{6 \sqrt{21}}{7}, \\
& l_{4}=\frac{150 \sqrt{3}}{49} \arcsin \left(\frac{1}{8}\right)-\frac{75 \sqrt{3}}{49} \pi-\frac{33 \sqrt{21}}{49} .
\end{aligned}
$$

Let $c_{1}(\delta)=0$, i.e., $a_{0}=0$; we have

$$
\begin{aligned}
c_{2}(\delta) & =\oint_{L_{1}} \frac{f(x)}{y} \mathrm{~d} x=\oint_{L_{2}} \frac{f(x)}{\sqrt{-2 A(x)}} \mathrm{d} x \\
& =2 \int_{0}^{9 / 7} \frac{f(x)-a_{0}}{\sqrt{-2 A(x)}} \mathrm{d} x .
\end{aligned}
$$

Direct computation gives

$$
c_{2}(\delta)=\sum_{i=1}^{9} J_{i} a_{i},
$$

where

$$
\begin{aligned}
& J_{1}=3 / 4 \sqrt{21}, \\
& J_{2}=2 \sqrt{3} \arcsin \left(\frac{1}{2}\right)-\frac{3}{4} \sqrt{21}+\sqrt{3} \pi, \\
& J_{3}=-\frac{12 \sqrt{3}}{7} \arcsin \left(\frac{1}{8}\right)+\frac{45 \sqrt{21}}{28}-\frac{6 \sqrt{3}}{7} \pi, \\
& J_{4}=\frac{150 \sqrt{3}}{49} \arcsin \left(\frac{1}{8}\right)-\frac{279 \sqrt{21}}{196}+\frac{75 \sqrt{3}}{49} \pi .
\end{aligned}
$$

Then, by (49) and (51) and Lemma 6, we have

$$
\widetilde{c}_{3}(\delta)=\sum_{i=1}^{9} \widetilde{J}_{i} a_{i},
$$

where $\widetilde{J}_{1}=J_{1}, \widetilde{J}_{i}=l_{i}+J_{i}, i=2,3, \ldots, 9$. 
Last we compute $b_{0}, b_{1}, d_{0}$, and $d_{1}$ in (36) and (37). Taking a linear transformation $u=4 \sqrt{30} / 49(x+3 / 7), v=y$ with time scaling $\mathrm{d} \tau=4 \sqrt{30} / 49 \mathrm{~d} t$ and applying Lemma 5 and program in [20], we obtain

$$
\begin{aligned}
& b_{0}=\sqrt{30} \pi\left(\frac{49}{60} a_{0}-\frac{7}{20} a_{1}+\frac{3}{20} a_{2}-\frac{9}{140} a_{3}+\frac{27}{980} a_{4}\right), \\
& b_{1}=\sqrt{30} \pi\left(\frac{939662563}{99532800} a_{0}-\frac{829307801}{165888000} a_{1}+\frac{254004191}{55296000} a_{2}-\frac{71782697}{18432000} a_{3}+\frac{17630543}{6144000} a_{4}\right) .
\end{aligned}
$$

Taking a linear transformation $u=\sqrt{40 / 7}(x-1), v=y$ with time scaling $\mathrm{d} \tau=\sqrt{40 / 7} \mathrm{~d} t$ and applying Lemma 5 and program in [20], we obtain

$$
\begin{aligned}
& d_{0}=\frac{1}{10} \pi \sqrt{70}\left(a_{0}+a_{1}+a_{2}+a_{3}+a_{4}\right), \\
& d_{1}=\frac{7 \sqrt{70}}{64000} \pi\left(327 a_{0}+111 a_{1}-25 a_{2}-81 a_{3}-57 a_{4}\right) .
\end{aligned}
$$

\section{Proof of Theorem 1}

In this section, we prove Theorem 1 . Solving the equations $c_{0}(\delta)=e_{0}(\delta)=c_{1}\left(S_{1_{\text {cusp }}}, \delta\right)=c_{1}\left(S_{2_{\text {saddle }}}, \delta\right)=0$ gives

$$
\begin{aligned}
& a_{0}=0, \\
& a_{1}=-\frac{27}{56} \\
& a_{2}=-\frac{27}{28}, \\
& a_{3}=\frac{29}{56} .
\end{aligned}
$$

We take $\delta=\left(a_{0}, a_{1}, a_{2}, a_{3}, 1\right)$ and $\delta_{0}=(0,-27 / 56,-27 /$ $28,29 / 56,1)$; then,

$$
\begin{aligned}
e_{3}\left(\delta_{0}\right) & =-\frac{3 \sqrt{3}}{196}\left(8 \pi-16 \arcsin \left(\frac{1}{8}\right)+15 \sqrt{7}\right) \\
& \approx-1.665254593<0, \\
c_{2}\left(\delta_{0}\right) & =-\frac{3 \sqrt{3}}{196}\left(-8 \pi-16 \arcsin \left(\frac{1}{8}\right)+15 \sqrt{7}\right) \\
& \approx-0.3326673051<0, \\
\tilde{c}_{3}\left(\delta_{0}\right) & =-\frac{3 \sqrt{3}}{98}\left(-16 \arcsin \left(\frac{1}{8}\right)+15 \sqrt{7}\right) \\
& \approx-1.997921898<0 .
\end{aligned}
$$

Therefore, there exist $h_{10}, h_{20}$, and $h_{30}$, satisfying $0<-h_{10} \ll 1,0<-h_{20} \ll 1$, and $0<h_{30} \ll 1$, respectively, such that

$$
\begin{aligned}
& M_{1}\left(h_{10}, \delta_{0}\right)=e_{3}\left(\delta_{0}\right) h_{10}+O\left(\left|h_{10}\right|^{7 / 6}\right)>0, \\
& M_{2}\left(h_{20}, \delta_{0}\right)=c_{2}\left(\delta_{0}\right) h_{20}+O\left(h_{20}^{2} \ln \left|h_{20}\right|\right)>0, \\
& M_{3}\left(h_{30}, \delta_{0}\right)=\widetilde{c}_{3}\left(\delta_{0}\right) h_{30}+O\left(h_{30}^{7 / 6}\right)<0,
\end{aligned}
$$

while taking $h=100$,

$$
\begin{aligned}
M_{3}\left(100, \delta_{0}\right) & =\oint_{L_{100}^{3}} f\left(x, \delta_{0}\right) y \mathrm{~d} x \\
& =\oint_{L_{100}^{3}} f\left(x, \delta_{0}\right) \sqrt{200-A(x)} \mathrm{d} x \\
& \approx 1678.486757>0,
\end{aligned}
$$

and thus there exists a $z_{30}$ between $h=h_{30}$ and $h=100$, such that

$$
M_{3}\left(z_{30}, \delta_{0}\right)=0
$$

It is easy to find

$$
\operatorname{Rank} \frac{\partial\left(c_{0}(\delta), e_{0}(\delta), c_{1}\left(S_{1_{\text {cusp }}}, \delta\right), c_{1}\left(S_{2_{\text {saddle }}}, \delta\right)\right)}{\partial\left(a_{0}, a_{1}, a_{2}, a_{3}\right)}=4
$$

Therefore, $e_{0}(\delta), c_{0}(\delta), c_{1}\left(S_{1_{\text {cusp }}}, \delta\right)$, and $c_{1}\left(S_{2_{\text {saddle }}}, \delta\right)$ can be taken as free parameters; we denote them by $e_{0}, c_{0}$, $c_{1}\left(S_{1_{\text {cusp }}}\right)$, and $c_{1}\left(S_{2_{\text {saddle }}}\right)$, respectively. Next, we take these free parameters to perturb 8 zeros.

We take $c_{1}\left(S_{2_{\text {saddle }}}\right), c_{1}\left(S_{1_{\text {cusp }}}\right), c_{0}, e_{0}$, in turn satisfying

$$
\begin{aligned}
& c_{1}\left(S_{2_{\text {saddle }}}\right)<0, \\
& c_{1}\left(S_{1_{\text {cusp }}}\right)>0, \\
& c_{0}>0, \\
& e_{0}<0, \quad 0<\left|e_{0}\right| \ll\left|c_{0}\right| \\
& \ll\left|c_{1}\left(S_{1_{\text {saddle }}}\right)\right| \ll\left|c_{1}\left(S_{2_{\text {cusp }}}\right)\right| \ll 1 .
\end{aligned}
$$

We take $\delta_{1}$ in $U_{1}\left(\delta_{0}, \epsilon\right) \triangleq\left\{\delta \mid e_{0}, c_{0}, c_{1}\left(S_{1_{\text {cusp }}}\right), c_{1}\left(S_{2_{\text {saddle }}}\right)\right.$ satisfying (62) $\}$ which is a very small neighborhood of $\delta_{0}$.

Therefore, $\quad e_{0}<0, \quad e_{1}>0, \quad e_{2}<0, \quad\left|e_{0}\right| \ll\left|e_{1}\right| \ll\left|e_{2}\right| \ll$ $\left|e_{3}\left(\delta_{0}\right)\right|$ for $M_{1}\left(h, \delta_{1}\right)$ by Lemma 6 ; then, there exist 3 zeros of $h_{11}, h_{12}$ and $h_{13}$ of $M_{1}\left(h, \delta_{1}\right)$ satisfying $0<-h_{13}<-h_{12}<$ $-h_{11}<-h_{10} \ll 1$.

Under (63), $\quad c_{0}>0, \quad c_{1}<0, \quad\left|c_{0}\right| \ll\left|c_{1}\right| \ll\left|c_{2}\left(\delta_{0}\right)\right|$ for $M_{2}\left(h, \delta_{1}\right)$ by Lemma 6 ; then, there exist 2 zeros $h_{21}, h_{22}$ of $M_{2}\left(h, \delta_{1}\right)$ satisfying $0<-h_{22}<-h_{21}<-h_{20} \ll 1$. 
Under (63), $\widetilde{c}_{0}>0, \widetilde{c}_{1}>0, \widetilde{c}_{2}<0,\left|\widetilde{c}_{0}\right| \ll\left|\widetilde{c}_{1}\right| \ll\left|\widetilde{c}_{2}\right| \ll \mid \widetilde{c}_{3}$ $\left(\delta_{0}\right) \mid$ for $M_{3}\left(h, \delta_{1}\right)$ by Lemma 6 ; then, there exist 3 zeros $h_{31}$, $h_{32}$, and $h_{33}$ of $M_{3}\left(h, \delta_{1}\right)$ satisfying $0<h_{33}<h_{32}<h_{31}<$ $h_{30} \ll 1$.

Note that

$$
M_{3}\left(h, \delta_{1}\right)=M_{3}\left(h, \delta_{0}\right)+O(\epsilon),
$$

and in accordance with (59), we obtain a $z_{30}^{*}$ near $z_{30}$, such that $M_{3}\left(z_{30}^{*}, \delta_{1}\right)=0$; therefore, there is another zero of $M_{3}\left(h, \delta_{1}\right)$ near $z_{30}$.

The above 9 zeros are isolated real zeros; therefore, they correspond to 9 limit cycles of system (6). The main theorem is proved.

\section{Conclusion and Discussion}

In the paper, we have applied the theories of Hopf bifurcation, homoclinic loop bifurcation, and heteroclinic loop bifurcation to detect the limit cycles near the center and polycycle locally. The main routine is to prove the independence of the coefficients of the asymptotic expansions of the Melnikov functions and then treat them as free perturbation parameters. The computational analysis shows that there exist at least 9 limit cycles in the suitably damped system. The result gives a relative larger lower bound on the number of limit cycles for the Liénard system of degree 5. It is interesting to show that the asymptotic expansions of the Melnikov functions not only are the efficient tools to detect limit cycles such as a complicated investigation in [24] but also have been successfully applied to study the existence of periodic traveling waves and the coexistence of periodic solitary traveling waves (see $[25,26])$. It may be more interesting to investigate the periodic traveling waves in external perturbation considered in the model given in [27]. It should also be pointed that there exist more questions left to solve for system (5), for example, what is the maximal number of limit cycles, or limit cycles by Poincaré bifurcation, or limit cycles by Hopf, or limit cycles by Hopf bifurcation, how about the global dynamics, and what is the period function of the undamped system. However, it is not easy at all to solve these questions.

\section{Data Availability}

The data used to support the findings of this study are available from the corresponding author upon request.

\section{Conflicts of Interest}

The authors declare that they have no conflicts of interest.

\section{Acknowledgments}

This study was supported by the Natural Science Foundation of Guangxi (2018GXNSFAA138198) and Program for Innovative Team of GUFE (2018-2021).

\section{References}

[1] M. Han and P. Yu, Normal Forms, Melnikov Functions and Bifurcations of Limit Cycles, Springer Science \& Business Media, Berlin, Germany, 2012.

[2] M. Han, Bifurcation Theory of Limit Cycles, Alpha Science International, London, UK, 2016.

[3] J. Li, "Hilbert's 16th problem and bifurcations of planar polynomial vector fields," International Journal of Bifurcation and Chaos, vol. 13, no. 1, pp. 47-106, 2003.

[4] M. Han and J. Li, "Lower bounds for the Hilbert number of polynomial systems," Journal of Differential Equations, vol. 252, no. 4, pp. 3278-3304, 2012.

[5] F. Dumortier and C. Li, "Perturbations from an elliptic Hamiltonian of degree four," Journal of Differential Equations, vol. 176, no. 1, pp. 114-157, 2001.

[6] F. Dumortier and C. Li, "Perturbations from an elliptic Hamiltonian of degree four," Journal of Differential Equations, vol. 175, no. 2, pp. 209-243, 2001.

[7] F. Dumortier and C. Li, "Perturbation from an elliptic Hamiltonian of degree four-III global centre," Journal of Differential Equations, vol. 188, no. 2, pp. 473-511, 2003.

[8] F. Dumortier and C. Li, "Perturbation from an elliptic Hamiltonian of degree four-IV figure eight-loop," Journal of Differential Equations, vol. 188, no. 2, pp. 512-554, 2003.

[9] J. Wang and D. Xiao, "On the number of limit cycles in small perturbations of a class of hyper-elliptic Hamiltonian systems with one nilpotent saddle," Journal of Differential Equations, vol. 250, no. 4, pp. 2227-2243, 2011.

[10] X. Sun and P. Yu, "Exact bound on the number of zeros of Abelian integrals for two hyper-elliptic Hamiltonian systems of degree 4," Journal of Differential Equations, vol. 267, no. 12, pp. 7369-7384, 2019.

[11] X. Sun and W. Huang, "Bounding the number of limit cycles for a polynomial Liénard system by using regular chains," Journal of Symbolic Computation, vol. 79, pp. 197-210, 2017.

[12] X. Sun and P. Yu, "Cyclicity of periodic annulus and Hopf cyclicity in perturbing a hyper-elliptic Hamiltonian system with a degenerate heteroclinic loop," Journal of Differential Equations, vol. 269, no. 11, pp. 9224-9253, 2020.

[13] A. Atabaigia and H. Zangeneha, "Bifurcation of limit cycles in small perturbations of a class of hyper-elliptic Hamiltonian systems of degree 5 with a cusp," Journal of Differential Equations, vol. 1, pp. 299-313, 2011.

[14] R. Kazemi, H. R. Z. Zangeneh, and A. Atabaigi, "On the number of limit cycles in small perturbations of a class of hyper-elliptic Hamiltonian systems," Nonlinear Analysis: Theory, Methods \& Applications, vol. 75, no. 2, pp. 574-587, 2012.

[15] X. Sun, "Exact bound on the number of limit cycles arising from a periodic annulus bounded by a symmetric heteroclinic loop," Journal of Applied Analysis \& Computation, vol. 10, no. 1, pp. 378-390, 2020.

[16] X. Sun, "Perturbation of a period annulus bounded by a heteroclinic loop connecting two hyperbolic saddles," Qualitative Theory of Dynamical Systems, vol. 16, no. 1, pp. 187203, 2017.

[17] X. Sun and L. Zhao, "Perturbations of a class of hyper-elliptic Hamiltonian systems of degree seven with nilpotent singular points," Applied Mathematics and Computation, vol. 289, pp. 194-203, 2016.

[18] Y. Xiong and M. Han, "New lower bounds for the Hilbert number of polynomial systems of Liénard type," Journal of Differential Equations, vol. 257, no. 7, pp. 2565-2590, 2014. 
[19] W. Xu and C. Li, "Limit cycles of some polynomial Liénard systems," Journal of Mathematical Analysis and Applications, vol. 389, no. 1, pp. 367-378, 2012.

[20] M. Han, J. Yang, and P. Yu, "Hopf bifurcations for nearHamiltonian systems," International Journal of Bifurcation and Chaos, vol. 19, no. 12, pp. 4117-4130, 2009.

[21] M. Han, J. Yang, A. A. Tarța, and Y. Gao, "Limit cycles near homoclinic and heteroclinic loops," Journal of Dynamics and Differential Equations, vol. 20, no. 4, pp. 923-944, 2008.

[22] M. Han, H. Zang, and J. Yang, "Limit cycle bifurcations by perturbing a cuspidal loop in a Hamiltonian system," Journal of Differential Equations, vol. 246, no. 1, pp. 129-163, 2009.

[23] X. Sun, M. Han, and J. Yang, "Bifurcation of limit cycles from a heteroclinic loop with a cusp," Nonlinear Analysis: Theory, Methods \& Applications, vol. 74, no. 9, pp. 2948-2965, 2011.

[24] Y. Xiong and M. Han, "A note on the expansion of the first order Melnikov function near a class of 3-polycycles," Journal of Differential Equations, vol. 2, pp. 125-130, 2020.

[25] X. Sun, P. Yu, and P. Yu, "Periodic traveling waves in a generalized BBM equation with weak backward diffusion and dissipation terms," Discrete \& Continuous Dynamical Systems-B, vol. 24, no. 2, pp. 965-987, 2019.

[26] X. Sun, W. Huang, and J. Cai, "Coexistence of the solitary and periodic waves in convecting shallow water fluid," Nonlinear Analysis: Real World Applications, vol. 53, 2020.

[27] J. Li, G. Chen, and J. Song, "Completing the study of traveling wave solutions for three two-component shallow water wave models," International Journal of Bifurcation and Chaos, vol. 30, no. 3, 2020 . 